\title{
Dermatopontin in the extracellular matrix enhances osteogenic differentiation of adipose-derived mesenchymal stem cells
}

\author{
Heather B. Coan ${ }^{1}$, Mark O. Lively ${ }^{2}$ and Mark E. Van Dyke ${ }^{3 *}$ \\ *Correspondence: mvandyk5@vt.edu

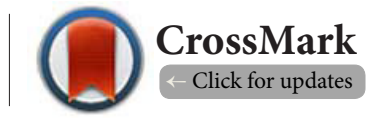 \\ 'Department of Biology, Western Carolina University, Cullowhee, NC 28723, USA. \\ ${ }^{2}$ Department of Biochemistry, Wake Forest School of Medicine, Winston-Salem, NC 27157, USA. \\ ${ }^{3}$ School of Biomedical Engineering and Sciences, Virginia Tech, Blacksburg, VA 24061, USA.
}

\begin{abstract}
Background: Dermatopontin (DPT) is a $22-\mathrm{kDa}$ tyrosine-rich extracellular matrix (ECM) protein that is found at high levels in demineralized bone matrix and cartilage and may play an important role in skeletal tissue function. In the current study, we investigate whether DPT in the ECM plays a role in the enhanced osteogenic differentiation of adipose-derived stem cells (ADSCs).

Methods: In order to determine whether DPT modulates osteogenesis, we overexpressed the DPT gene in ADSC using stable lentiviral infection, induced the DPT-overexpressing cells to differentiate, and isolated the ECM secreted by these cells during osteogenesis. Using the secreted ECM with "higher than normal" levels of DPT embedded within as a substrate for cell growth, we assessed the extent to which the excess DPT modulated osteogenic marker expression during osteogenic differentiation of naive ADSC.

Results: We found that ADSC cultured on the DPT-enriched ECM differentiated towards an osteogenic phenotype more robustly, as measured by expression of osteogenic marker genes.

Conclusions: This may indicate an important role for DPT in the induction of stem cells toward an osteoblast phenotype during skeletal wound healing. DPT may present a novel candidate for future studies of stem cell osteogenesis and the development of more biologically relevant biomaterial substrates for bone regeneration.
\end{abstract}

Keywords: Dermatopontin, adipose-derived stem cells, extracellular matrix, osteogenesis, overexpression, matricellular proteins

\section{Introduction}

Dermatopontin (DPT) is a 22-kDa tyrosine-rich extracellular matrix (ECM) protein that is found at high levels in demineralized bone matrix and cartilage and may play an important role in skeletal tissue function [1-3]. It is thought that DPT acts largely as a mediator between the cell and the ECM environment $[1,4,5]$. Functional roles attributed to DPT with relevance to bone include binding and modulation of bone morphogenetic protein 2 (BMP2) and transforming growth factor $b$ (TGFb), cell adhesion, collagen and fibronectin fibril formation, cell proliferation, and reported activity as a downstream target of the vitamin D receptor (VDR) [1,3,4,6-9].

In a previous study, we identified the DPT gene as being differentially expressed during adipose-derived stem cell
(ADSC) osteogenic differentiation [10]. We demonstrated that matrices secreted by ADSC at the midpoint in a 30-day cell culture differentiation protocol enhanced osteogenesis. Interestingly, we found that matrices secreted at earlier stages in differentiation appeared to inhibit osteogenesis. Comparison of genes that coded for extracellular matrix proteins in cells that secreted osteogenesis-inhibiting matrices versus cells that secreted osteogenesis-promoting matrices revealed significant upregulation of several genes with potential roles in osteogenesis, including DPT, in cells that secreted the osteogenesis-promoting matrices.

In the current study, we investigate whether DPT in the ECM plays a role in the enhancedosteogenic differentiation of ADSC. We hypothesized that a DPT enriched micro environment 
Coan et al. Musculoskeletal Biology 2014,

would enhance osteogenesis of ADSC. In order to determine whether DPT modulates osteogenesis, we overexpressed the DPT gene in ADSC using stable lentiviral infection, induced the DPT-overexpressing cells to differentiate, and isolated the ECM secreted by these cells during osteogenesis. Using the secreted ECM with "higher than normal" levels of DPT embedded within as a substrate for cell growth, we assessed the extent to whichthe excess DPT modulated osteogenic marker expression during osteogenic differentiation of naive ADSC. Our results indicate that DPT may be a novel osteogenesisstimulating matricellular protein.

\section{Materials and methods}

\section{Adipose-derived stem cell isolation and culture}

Human ADSC were obtained, isolated, and characterized as previously described [10] under a protocol approved by the Wake Forest School of Medicine Institutional Review Board (no. IRB00007586). ADSC were maintained in adipose maintenance media (AMM) consisting of Dulbecco's Modified Eagle Medium (DMEM) high glucose (Gibco, Invitrogen) supplemented with $10 \%$ heat inactivated fetal bovine serum (FBS) (Gibco) and 1\% Antibiotic/Antimycotic (A/A) solution (Sigma). Plastic adherent ADSC were maintained in $\mathrm{AMM}$ at $37^{\circ} \mathrm{C}$ and $5 \% \mathrm{CO}_{2}$.

Three ADSC cell lines were established from three different donors. All cell lines were used in the experiments as individual samples and analyzed together as replicates. Experiments were performed using cells at passage 2 .

Western blot confirmation of higher DPT protein expression in ADSC at the midpoint in differentiation versus ADSC at earlier in differentiation

Our previous work demonstrates that ADSC at the midpoint in osteogenesis secrete osteogenesis-enhancing ECM [10]. Alternatively, ADSC at an earlier time point in differentiation secrete osteogenesis-inhibiting ECM. In this study, we hypothesized that genes coding for ECM proteins that were highly expressed by cells at the midpoint in osteogenesis versus cells from an earlier time point might represent components of the ECM responsible for the promotion of osteogenesis. We used western blot analysis to confirm high DPT protein expression with our previously observed increased DPT mRNA expression in ADSC at the midpoint in differentiation (day 16: secretes osteogenesis-promoting matrices) versus ADSC at an earlier time point (day 10: secretes osteogenesis-inhibiting matrices).

We collected protein preps from ADSC at day 16 and 10 in osteogenic differentiation. Protein preps were prepared by first washing plates with PBS to remove unbound cells and protein. Cell lysis and protein solubilization with a detergent

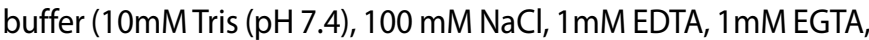
$1 \%$ Triton X-100, $10 \%$ glycerol, $0.1 \%$ SDS, $0.5 \%$ deoxycholate, complete ULTRA Protease Inhibitor Tablet (Roche Diagnostics)) were performed directly on the dish with shaking for $1 \mathrm{hr}$. After incubation with shaking, the resulting lysate and ECM prep were scraped off of the dish. New detergent buffer was added for a second round of incubation and scraping in order to remove loosely adhered proteins. It should be noted that due to the insoluble nature of ECM proteins, some protein was left on the dish following scraping. However, because all samples are treated in the same manner, this amount should be similar across control and experimental groups. Protein concentration was determined using a Protein Determination Modified Lowry kit (Sigma-Aldrich). The solubilized protein was loaded at equal concentrations onto SDS-PAGE, Tris-HCl 12.5\% gels and separated by relative size. Separated proteins were transferred to Immobilon P membranes (Millipore Corporation) and the membranes were probed with primary antibodies for DPT (Novus Biologicals) and GAPDH (loading control, AbCam). Secondary horseradish peroxidase-conjugated antibodies (Roche Molecular Biochemicals) were used and protein bands were visualized with a chemiluminescence detection system (ECL lit; Amersham).

Lentiviral-mediated overexpression of dermatopontin After confirming that DPT is highly expressed at the midpoint in osteogenesis, we designed a lentiviral dermatopontin construct that was used to achieve stable overexpression of DPT in ADSC. The experimental design following infection with the lentiviral construct is depicted in Figure 1.

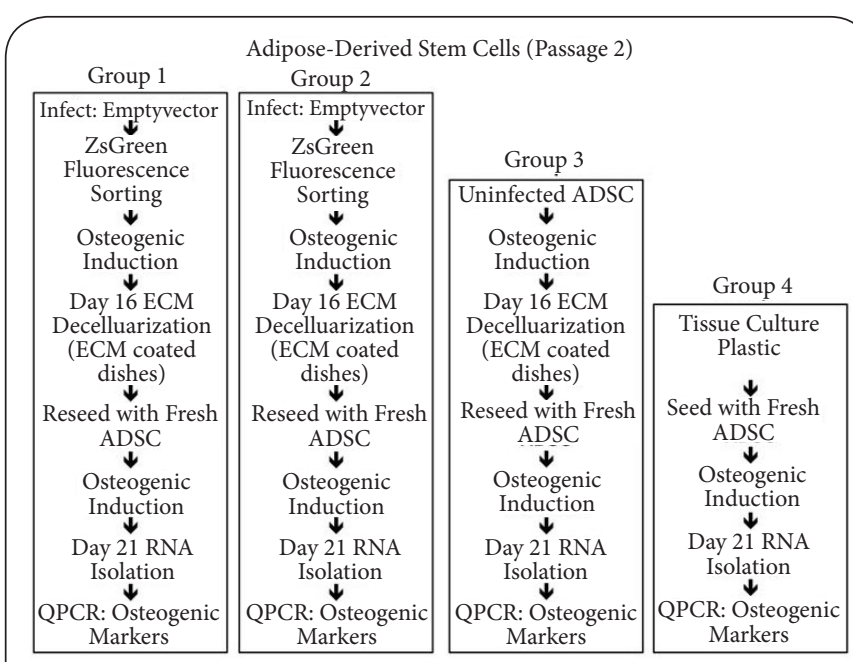

Figure 1. Experimental design.

\section{Construction of the recombinant lentivirus}

A clone containing the DPT gene was obtained from Invitrogen (MGC Full Length (IRAT) Human Dermatopontin Clone) in the form of plasmid DNA contained in a bacterial carrier (pCMVSPORT6). The plasmid DNA was expanded in bacterial culture and subsequently isolated using QIAGEN's Plasmid Maxi Prep kit according to the manufacturer's instructions. The lentivirus vector, pLVX-EFLa-IRES-ZsGreen1, was obtained from Clontech and used to create a lentivirus DPT green fluorescent vector construct (Lenti_DPT) by cutting the DPT gene out of the 
plasmid vector using restriction digests (EcoRI \& Xbal, New England BioLabs) and subsequently ligating DPT into the cut lentivector using T4 DNA Ligase (New England BioLabs). The Lenti_DPT construct was transformed into TOP10 E. coli cells (Life Technologies), expanded and isolated with bacterial culture and subsequent plasmid isolation (QIAGEN, Plasmid Mini Prep kit).

Human embryonic kidney 293T (HEK293T) cell culture HEK293T cells were obtained from Invitrogen. Cells were maintained in DMEM supplemented with $10 \%$ FBS, $1 \%$ L-glutamine, and $1 \%$ penicillin/streptomycin (P/S)(Sigma).

Transfection of HEK293T cells to produce virus

HEK293T cells were plated onto gelatin-coated dishes at a density of $1.3 \times 10^{4}$ cells $/ \mathrm{cm}^{2}$ and allowed to grow overnight to approximately $80 \%$ confluence. Cells were transfected using FuGene HD (Roche) according to the manufacturer's instructions. Briefly, a packaging vector (psPAX2)(Clontech), an envelope vector (pMD2.G)(Clontech), and the Lenti_DPT vector were added to DMEM unsupplemented at a ratio of 1:2:3 (packaging: envelope: Lenti_DPT), respectively. A control group was also created by transfecting HEK293T cells with the empty lentiviral vector(Empty_vector) (without the DPT gene insert). FuGene HD was added to the DMEM vector solution at a ratio of 4:1 (FuGene: vector). The vector/FuGene HD mixture was incubated for 20 minutes. HEK293T cell media was switched to antibiotic free HEK293T cell media and the vector/FuGene HD mix was added dropwise to the cells. Cells were maintained in transfection media overnight. The transfection efficiency was monitored using fluorescence microscopy (Zeiss, Axiovert). Viral supernatants were collected 48 and 72 hours post transfection, combined, filtered through a $0.45 \mu \mathrm{m}$ filter, and stored at $-80^{\circ} \mathrm{C}$ until further use.

Infection of ADSCs with viral supernatants

ADSC were plated at a density of $1 \times 10^{4} \mathrm{cell} / \mathrm{sm}$ and allowed to grow overnight to approximately $60-70 \%$ confluence. Cells underwent multiple infections (three total) with the virus-containing-medium, either Lenti_DPT medium or Empty_vector medium, at a ratio of 1:5 (virus medium: AMM) in the presence of $8 \mathrm{ng} / \mathrm{mL}$ polybrene (1,5-dimethyl1,5-diazaundecamethylene polymethobromide, Sigma). A progressive increase in infected cells was observed following each infection (as monitored through fluorescence). After the third infection, cells were sorted using ZsGreen fluorescence (excitation $493 \mathrm{~nm}$, emission $505 \mathrm{~nm}$ ) as the selective agent (FACSAriall, BD Biosciences). Quantitative real-time polymerase chain reaction (QPCR) was used to determine whether DPT mRNA expression increased in the Lenti_DPT infected cells that were positive for ZsGreen versus the cells that were negative for ZsGreen and the cells that were infected with the Empty_vector. Subsequent experiments were performed on the sorted ZsGreen fluorescing cells infected either with the Lenti_DPT construct or Empty_vector.

\section{Confirmation of overexpression}

Stable overexpression of DPT was confirmed using QPCR and western blot analysis. Expression of DPT in ADSC infected with the Lenti_DPT vector was compared to DPT expression in ADSC infected with the Empty_vector and uninfected ADSC at the midpoint in osteogenic differentiation. The midpoint was previously shown to have high levels of DPT mRNA and secretion of osteogenesis-promoting matrices.

QPCR analysis of DPT gene expression in the infected cells

To determine whether Lenti_DPT infected cells overexpressed the DPT gene following cell sorting, we extracted total RNA from ZsGreen (+) Lenti_DPT infected ADSC, ZsGreen (-) Lenti_DPT infected ADSC, and ZsGreen (+) Empty_vector infected ADSC immediately after cell sorting. RNA was extracted using the Perfect Pure RNA Cell Culture Kit according to the manufacturer's instructions (5 Prime). Extracted RNA was quantified using a Thermo Scientific NanoDrop spectrophotometer and checked for integrity with gel electrophoresis. RNA was transcribed into CDNA using oligo (dT) primers and SuperScript II reverse transcriptase (Invitrogen). Taqman Universal PCR Master Mix Kit (Life Technologies) was used with $0.5 \mu \mathrm{g}$ total RNA for each reaction. Reactions were performed using an Applied Biosystems ABI 7300 Real-Time PCR System. Primers for QPCR were obtained from Life Technologies and included 18S RNA (Hs03003631_g1, the endogenous control) and DPT (Hs00355356_m1). Relative expression of these genes was determined following normalization to the level of the $18 S$ RNA housekeeping gene, according to the method of Livak and Schmittgen [11].

Western blot analysis of DPT protein overexpression Western blot analyses were used to verify that the Lenti_DPT infected cells expressed higher protein levels of DPT compared to the uninfected cells and the Empty_vector infected cells. Protein preps were collected from Lenti_DPT infected cells at the midpoint in osteogenesis. Protein preps were also collected from the Empty_vector infected ADSC and from uninfected ADSC at the midpoint in osteogenesis. Protein preparations, concentration determination, SDS-PAGE, and western blotting were performed as described above. Note that overexpression was measured in cells and ECM as one sample to show increased DPT within overexpressing cultures. Due to a limited yield of DPT-overexpressing cells that prohibited isolation of excessive quantities of ECM for western blot troubleshooting and due to alack of an appropriate endogenous ECM loading control protein, we isolated preps from both cells and ECM combined, rather than from only the ECM. For this reason, the amounts present in the western blot analyses do not necessarily reflect total DPT within the ECM. Although DPT is a secreted protein, prior to 
Coan et al. Musculoskeletal Biology 2014,

protein secretion, cells would be expected to also contain DPT. Therefore, the total DPT shown reflects that which was present in the cells as well as that which was scrapped off of the dish and solubilized.

\section{Osteogenic differentiation}

Bone differentiation of ADSC was performed according to the method of Zuk et al., with minor adjustments [12]. Lenti_DPT infected cells, Empty_vector infected cells, and uninfected cells were all induced to differentiate. Briefly, ADSC were plated onto tissue culture dishes at $3000 \mathrm{cells} / \mathrm{cm}^{2}$. Cells underwent a "pre-induction" growth period of approximately 2 days until they reached $\sim 70 \%$ confluence in AMM. Bone media consisting of DMEM low-glucose (Gibco, Invitrogen) supplemented with $10 \% \mathrm{FBS}, 1 \% \mathrm{AA}$, and osteogenic supplements $100 \mathrm{nM}$ dexamethasone (Sigma), $10 \mathrm{mM}$ b-glycerophosphate (Calbiochem), and $0.05 \mathrm{mM}$ ascorbic acid-2-phosphate (Sigma) was added following the pre-induction growth period. Media was changed every 3-4 days.

ECM isolation and preparation for use as a growth substrate

ECM was isolated from Lenti_DPT infected ADSC, Empty_vector infected ADSC, and uninfected ADSC undergoing osteogenic differentiation at the midpoint in osteogenesis (day 16). Detergent lysis of the cells and removal of cellular components was performed according to published methods $[13,14]$. Briefly, cultures were washed with phosphate buffered saline (PBS). PBS supplemented with $0.5 \%$ Triton X-100 and $20 \mathrm{mM}$ $\mathrm{NH}_{4} \mathrm{OH}$ was added and allowed to incubate approximately 5 minutes at $37^{\circ} \mathrm{C}$. After visual confirmation of cell release, ECM coated dishes were incubated with $100 \mu \mathrm{g}$ DNAse I and 100 $\mu \mathrm{g}$ RNAse A (5 Prime) for 1 hour at $37^{\circ} \mathrm{C}$. ECM coated dishes were washed $2 \mathrm{X}$ with $\mathrm{PBS}$ and stored at $4^{\circ} \mathrm{C}$ in $1 \%$ AA spiked PBS (no longer than 1 week) until further use.

\section{Cell seeding onto ECM growth substrates}

Undifferentiated ADSC were seeded onto the secreted ECMcoated culture dishes and induced to differentiate with bone medium. The ECM substrates used for cell seeding included osteogenic ECM derived from Lenti_DPT infected ADSC, Empty_vector infected ADSC, and uninfected ADSC. ADSC were also seeded onto tissue culture plates (TCP control) and induced to differentiate as an uncoated control. The seeded cells were allowed to differentiate for 21 days on the ECM substrates and TCP with media changes every 3-4 days.

QPCR analysis of osteogenic markers

QPCR analyses of cells seeded onto the ECM substrates and the TCP control were performed as described above. Primers were obtained from Life Technologies and included the $18 \mathrm{~S}$ RNA housekeeping gene, runt-related transcription factor 2 (RUNX2, Hs01047978_m1), osteocalcin (OCN, Hs0060945_g1), WW domain containing transcription regulator 1 (TAZ, HsO- 0210007_m1), and wingless-type MMTV integration site family, member 5A (WNT5A; Hs00998537_m1). Expression levels were analyzed relative to the 18S RNA control and all samples were normalized and presented as fold changes over undifferentiated ADSC gene levels.

\section{Statistical analyses}

Statistical analyses were performed on the QPCR data. Duplicate samples (one sample=one well of a 6-well dish) from each of three donor samples were used to calculate means and standard deviations (SD) for each parameter (i.e., 3 donors $x 2$ replicates $=6$ data points). GraphPad Prism software was used for standard $t$-tests as well as analyses of variance (ANOVA) to compare groups and to determine significance of the crosswise comparisons. Multiple group comparisons were analyzed using One Way ANOVA and Tukey's Multiple Comparison post hoc analysis.

\section{Results}

DPT protein levels in ADSC induced to differentiate on TCP were analyzed in order to determine whether previously observed high DPT mRNA expression correlated with high protein levels at the midpoint in osteogenesis. Faint DPT antibody banding was observed at day 10 in osteogenesis, the time point at which secreted osteogenesis-inhibiting matrices were present. However, prominent DPT bands near the expected $22 \mathrm{kD}$ size were observed in all replicates at day 16 of osteogenesis, the day at which secreted osteogenesis-promoting matrices were present (Figure 2). The GAPDH loading controls indicated that similar protein levels were loaded onto the gels.

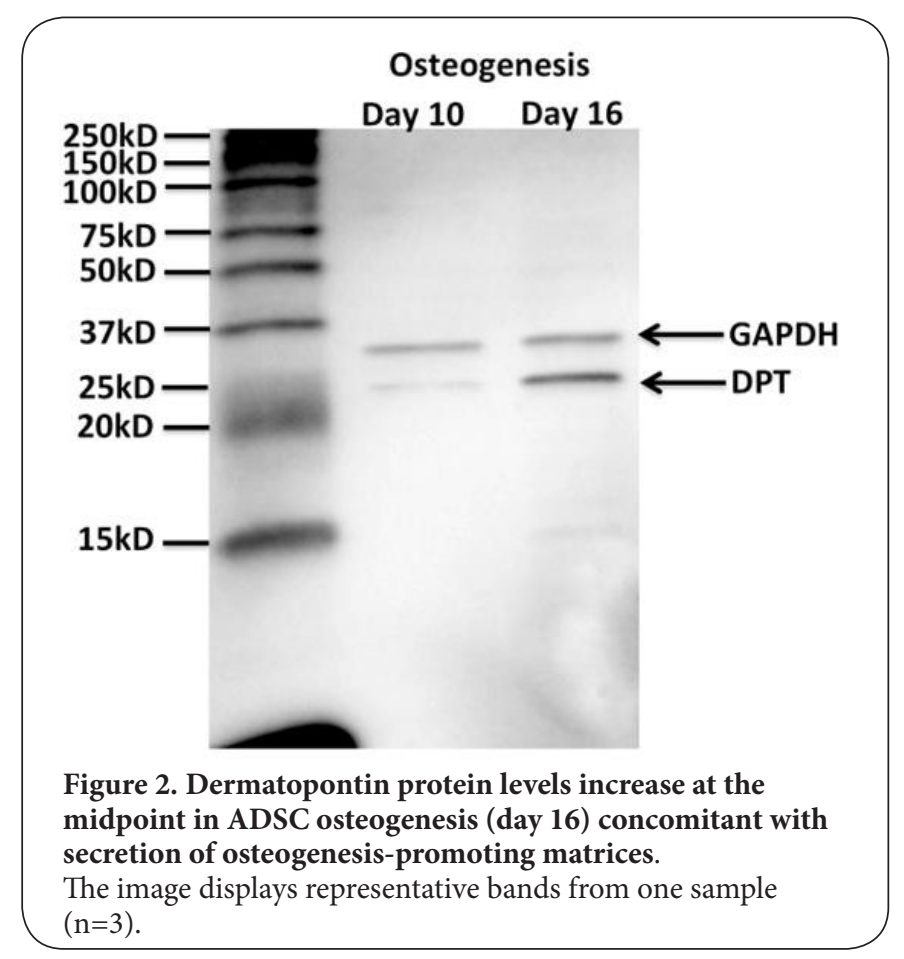


After infecting ADSC cultures with the Lenti_DPT construct and sorting for ZsGreen fluorescence, increased DPT gene expression was observed in the ZsGreen (+) Lenti_DPT cells versus the ZsGreen (-)cells and the ZsGreen (+) Empty_vector cells using QPCR (Figure 3A). Cells that sorted negative for ZsGreen expressed low levels of DPT suggesting that the Lenti DPT vector did not integrate itself into the genome. Similarly, cells that sorted positive for ZsGreen in the Empty_vector infected cells also expressed low levels of DPT suggesting that the empty vector did integrate itself, but only contained the ZsGreen gene and not the DPT gene. In contrast, cells that sorted positive for ZsGreen in the Lenti_DPT infected cells expressed significantly higher levels of DPT showing that the Lenti_DPT vector containing both the DPT gene and the ZsGreen gene integrated into the cells.

In addition to high gene expression, ADSC cultures infected with the Lenti_DPT vector contained higher levels of the DPT protein (Figure 3B). Because the presence of DPT in the ECM at day 16 during osteogenesiswas expected to correlate with an increase in the capacity of the matrices to enhance osteogenesis, DPT protein levels at day 16 during osteogenesis were compared across treatment groups. Specifically, Lenti_DPT infected cells at day 16 in osteogenesiscontained higher levels of DPT than Empty_vector infected cells and uninfected cells at day 16 in osteogenesis.

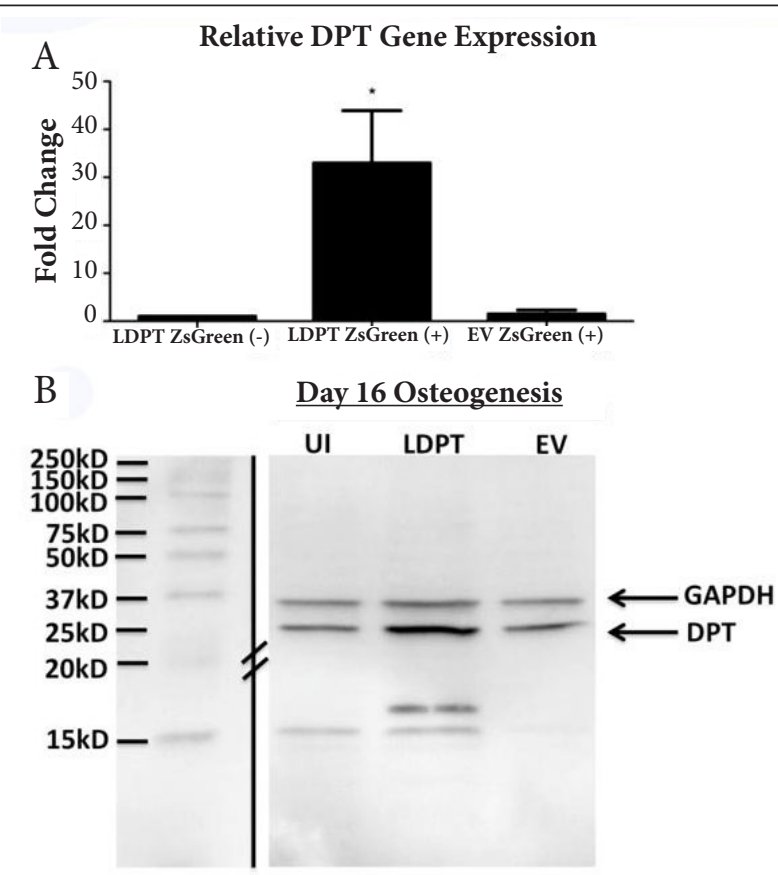

Figure 3. Dermatopontin overexpression was confirmed by QPCR and western blot. A) mRNA was quantified relative to 18 S RNA. Bars represent mean \pm SD of 6 replicates normalized to ZsGreen (-) cells ( ${ }^{*} \mathrm{p} \leq 0.05, \mathrm{LDPT}=$ Lenti_DPT, EV $=$ Empty vector). B) Dermatopontin increases in Lenti_DPT infected compared to uninfected (UI) and Empty_vector infected cells at osteogenesis day 16 (standard ladder in far left lane).
Expression of osteogenic marker genes (RUNX2, OCN, and TAZ) was analyzed in ADSC seeded onto the different ECM coatings (Figure 4). Expression of the WNT5A signaling protein was also analyzed. Comparisons were made between ADSC induced to differentiate on:1) ECM secreted at the midpoint in osteogenesis containing higher than normal levels of DPT (secreted by Lenti_DPT infected cells); 2) ECM secreted at the midpoint in osteogenesis by uninfected ADSC (the time point that secretes osteogenesis-promoting matrices); 3) ECM secreted at the midpoint inosteogenesis by Empty_vector infected cells (expected to be the same composition as ECM secreted from uninfected cells unless the virus integrated into a genomic region that affects osteogenesis, only RUNX2 and OCN expression were analyzed in this group due to small RNA yields); and 4) TCP alone. Expression analyses revealed significantly higher levels of RUNX2, OCN, and TAZ in ADSC cultured on ECM enriched with secreted DPT (DPT_ECM) compared to all other groups. As observed previously, ADSC seeded onto matrices from the midpoint in differentiation (ECM secreted by uninfected cells and Empty_vector infected cells), in general, also displayed enhanced osteogenic gene expression compared to the TCP seeded cells, but lower expression compared to cells on DPT_ECM. WNT5A gene expression was not statistically different between any of the groups, although the expression trend observed indicates that ADSC seeded onto both ECM coatings (DPT_ECM and "normal" ECM) express higher levels of WNT5A than ADSC on TCP.
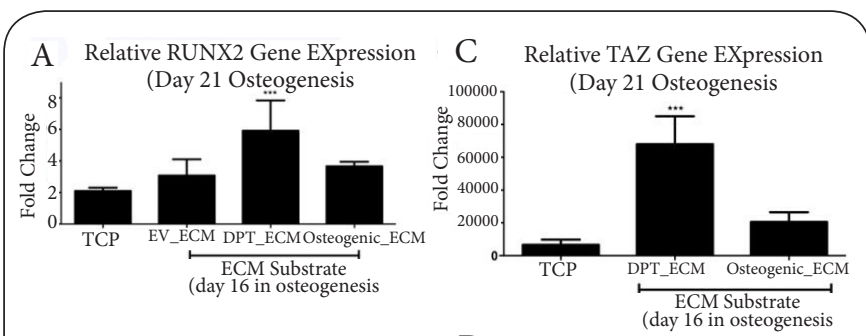

B Relative Osteocalcin Gene EXpression D Relative Wnt5A Gene EXpression
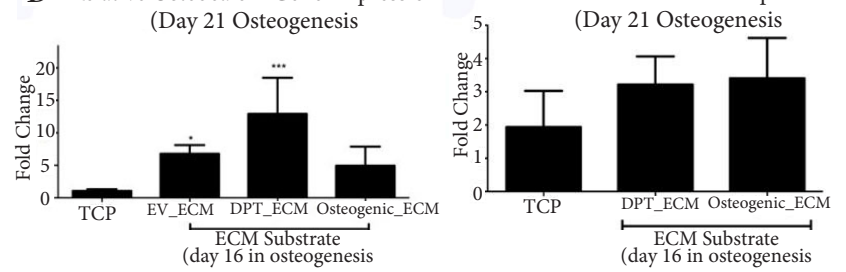

Figure 4. Gene expression of A) RUNX2, B) OCN, C) TAZ, and D) WNT5A increases in ADSC seeded onto osteogenic matrices containing abundant DPT. mRNA was quantified relative to $18 \mathrm{~S}$ RNA. Bars represent mean \pm SD of 6 replicates normalized to undifferentiated ADSC $\left({ }^{*} \mathrm{p} \leq 0.05\right.$ and ${ }_{* * *} \mathrm{p} \leq 0.001$ compared to the TCP control).

We also performed gene expression analyses in DPT-overexpressing cells induced to undergo osteogenic differentiation on TCP alone. We found that the expression of all osteogenic markers was not significantly different than that of ADSC 
Coan et al. Musculoskeletal Biology 2014,

grown on TCP alone, without an ECM substrate.

\section{Discussion}

It is widely understood that growth factors are important in osteogenesis. However, skeletal ECM has also been shown to have a beneficial effect on osteogenesis. Much of the published work concerning the effect of individual ECM components on osteogenesis has centered on the more abundant matrix proteins and on inorganic ECM components common in mineralized tissue [15-19]. Also commonly studied are the influences of synthetic ECM-mimicking substrates and various growth factors [15,20-22]. However, small, non-collagenous, matricellular ECM components may have equally or more important roles in modulating the differentiation process and are much less studied. For this reason, we selected DPT, a small extracellular protein highly expressed at the gene and protein levels at the midpoint in ADSC osteogenesis [10], for overexpression studies to determine its effect on osteogenesis.

Our previous work [10] suggests that ADSC secrete osteogenic matrices at day 16 during differentiation. To determine potential proteins responsible for this enhanced activity, we identified genes that code for ECM components that were upregulated at day 16 in ADSC differentiation - one of which was DPT. We chose DPT for further study based on previous work suggesting a role for DPT in wound healing and in cellmatrix interaction coordination.

However,we observed increased expression at this time point of a number of ECM-related genes, all --or many-- of which may also be involved in promoting osteogenesis. Of specific interest, was DPT's role as a potential matricellular protein. Our results show that DPT only enhances osteogenesis in the presence of other ECM proteins, but not in DPT-overexpressing cells grown on TCP. This could be explained by the potential matricellular nature of DPT and by our previous findings that show that the ECM is extremely important to differentiation. Matricellular proteins do not typically have biological effects unless they are in the presence of appropriate cell-matrix components. As a matricellular protein, DPT would not be expected to show activity unless the correct matrix components in a specific tertiary/quaternary structure are present, which we observed in our study. Further studies will be important for determining exactly how DPT interacts with the matrix and cells to promote differentiation.

Matricellular proteins are small, insoluble ECM components that do not provide structure or stabilization for cells and tissues, but rather regulate cell-related processes such as adherence, signaling, and growth factor binding by modulating cellmatrix interactions [23-27]. These proteins are often key accessory proteins involved in cell-ECM interactions and growth factor and cytokine binding [24,25], and although they do directly modulate cell behavior, they can have profound indirect effects on cells by promoting growth factor and cytokine signaling $[\mathbf{2 4 , 2 5 ]}$. Matricellular proteins: 1 ) are often expressed at high levels during developmental processes and in response to injury; 2 ) are not structural proteins; 3 ) bind surface receptors, cytokines, growth factors, and ECM proteins; 4) do not typically enhance adhesion; and 5) when targeted for gene disruption do not result in overt phenotypic changes unless the cell is responding to injury or undergoing development $[\mathbf{2 4 , 2 5 ]}$. Importantly, matricellular proteins are highly expressed during early embryonic development; however, in adults, altered expression of these proteins does not significantly effect healthy cells or tissue, only cells and tissues undergoing wound healing or remodeling $[24,25]$. This indicates that matricellular proteins may be vital to healing and regeneration of wounded tissue and that by leveraging the endogenous response to these proteins, we may be able to leverage wound healing capacity without altering healthy tissue behavior, which is a common and negative side effect of osteoinductive BMPs that induce bone formation even in healthy tissue.

Although DPT has not been classified specifically as a matricellular protein, most of its previously identified characteristics fit with the five characteristics of matricellular proteins. Most convincingly, DPT modulates cell behavior indirectly by modulating cell-ECM interactions [1], DPT binds several known growth factors including TGF $\beta$ and BMP2 $[3,4]$, altered DPT expression is observed during tissue injury [28-33], and DPT knockout mice only show subtle changes to skin elasticity [7]. In addition, Okamoto \& Fujiwara suggest that DPT contributes predominately to matrix remodeling during wound healing and that further studies on wound healing in DPT transgenic mice mayeluci date the specific roles of DPT in response to injury [1].

The enhanced osteogenesis that we observed in ADSC cultured on ECM containing abundant DPT may reflect the ability of DPT to alter the extracellular environment to promote wound healing and/or development. We did not observe enhanced osteogenesis in DPT-overexpressing ADSC induced to differentiate on TCP. In these cultures, DPT would be found, both within the conditioned culture medium as well as, at later time points in differentiation, within ECM deposited on the dish. The lack of enhanced osteogenesis in these cells however, suggests that DPT within the conditioned medium does not act to promote early osteogenic induction. Rather, enhanced osteogenesis was only observed in cells seeded onto DPT_ECM, further supporting the notion that DPT acts in concert with other ECM components to modulate ADSC osteogenesis through cell-ECM interactions.

As previously determined, matricellular proteins have fairly benign roles during healthy tissue function $[\mathbf{2 4 , 2 5 ]}$; however, high expression following tissue injury appears to "jump start" a cascade of extracellular responses to injury $[23,24]$. Mesenchymal stem cell differentiation can be viewed as: 1 ) as a recapitulation of early developmental processes and 2) as a means of regenerating tissue following injury $[15,34]$. Both processes are highly affected by altered matricellular expression [23-25]; therefore, our results indicate that DPT may 
play a vital role in skeletal wound healing as a matricellular protein.

Our results show that both of the groups cultured on ECM (DPT enriched and osteogenic ECM) had increased expression of WNT5A compared to those grown on TCP, but the differences were not significant.The increased expression trend may be relevant due to recent advances in our understanding of how cells in culture behave in response to their microenvironment. Specifically, the calcium-dependent Wnt signaling pathway, which WNT5A is a part of, is gaining recognition as a pathway involved in cell differentiation and behavior when cells are cultured on microstructured surfaces $[20,35]$.

In addition to our findings of DPT's osteogenic activity, we also present a novel method for investigating cell response to extracellular matrix in culture. Our approach of genetically modifying ADSC to secrete DPT into the endogenous ECM allowed us to increase DPT content within the ECM without removing potential accessory ECM components. Rather than simply coating dishes with DPT, we were able to create a more natural ECM environment with incorporated DPT. To our knowledge, this approach has not been previously utilized and represents a novel method for investigating overexpressed ECM protein affect on cell behavior and differentiation. In particular, the approach is especially important for studies that are aimed at investigating matricellular proteins, due to their interactions with ECM proteins. Without the native ECM, studies that focus on cell response to matricellular proteins may not provide an accurate portrayal of how the protein would affect cell behavior.

The results reported here suggest that DPT can induce significant up-regulation of osteogenic markers in vitro. This may indicate an important role for DPT in the induction of stem cells toward an osteoblast phenotype during skeletal wound healing. Further studies are needed to determine the mechanism of action. However, this study provides a first pass at understanding the complexities of cell-matrix interactions. Our culture system that utilizes cell-secreted proteins in addition to an overexpressed matricellular protein may be a system more suited for studying matricellular proteins and their effects on cell behavior. Future studies will focus on the mechanism by which DPT promotes osteogenesis within the ECM.

\section{Conclusions}

Stem cell-secreted osteogenic ECM guides naïve stem cells toward specific lineage determination targets. This study follows on earlier work that used gene array to identify potential gene products that provide microenvironmental cues to differentiating ADSC. Our present findings suggest that enrichment of the cell-secreted ECM in specific proteins through a novel genetic engineering approach can further enhance lineage specification and improve the efficiency of stem cell differentiation protocols. Broadly applied, these techniques can be used to discover important signaling molecules in a multitude of differentiation systems that can serve as targets for biomaterial development efforts for tissue engineering and regenerative medicine.

List of abbreviations

DPT: Dermatopontin

ADSC: Adipose-derived stem cells

ECM: Extracellular matrix

BMP2: Bone Morphogenetic Protein 2

TGFb: Transforming Growth Factor $b$

VDR: Vitamin D receptor

AMM: Adipose Maintenance Media

DMEM: Dulbecco's Modified Eagle Medium

Competing interests

The authors declare that they have no competing interests.

Authors' contributions

\begin{tabular}{|l|c|c|c|}
\hline Authors' contributions & HBC & MOL & MVD \\
\hline Research concept and design & $\checkmark$ & $\checkmark$ & $\checkmark$ \\
\hline Collection and/or assembly of data & $\checkmark$ & -- & -- \\
\hline Data analysis and interpretation & $\checkmark$ & $\checkmark$ & $\checkmark$ \\
\hline Writing the article & $\checkmark$ & -- & $\checkmark$ \\
\hline Critical revision of the article & $\checkmark$ & $\checkmark$ & $\checkmark$ \\
\hline Final approval of article & -- & -- & $\checkmark$ \\
\hline Statistical analysis & $\checkmark$ & -- & -- \\
\hline
\end{tabular}

Acknowledgement

The authors thank Chris Booth for assistance with cell culture, and Drs. David Mack and Emily Moorefield for helpful discussions related to the experimental protocols used in this study. This study was funded in part by a grant from the Department of Energy (award no. DE-FG02-06ER64240).

Publication history

Editor: Paul Park, University of Michigan, USA.

Received: 27-Feb-2014 Final Revised: 23-Jul-2014

Accepted: 06-Aug-2014 Published: 25-Aug-2014

\section{References}

1. Okamoto $\mathrm{O}$ and Fujiwara S. Dermatopontin, a novel player in the biology of the extracellular matrix. Connect Tissue Res. 2006; 47:177-89. | Article | PubMed

2. Forbes EG, Cronshaw AD, MacBeath JR and Hulmes DJ. Tyrosine-rich acidic matrix protein (TRAMP) is a tyrosine-sulphated and widely distributed protein of the extracellular matrix. FEBS Lett. 1994; 351:4336. I Article | PubMed

3. Behnam K, Murray SS and Brochmann EJ. BMP stimulation of alkaline phosphatase activity in pluripotent mouse $\mathrm{C2C12}$ cells is inhibited by dermatopontin, one of the most abundant low molecular weight proteins in demineralized bone matrix. Connect Tissue Res. 2006; 47:271-7. | Article | PubMed

4. Okamoto O, Fujiwara S, Abe M and Sato Y. Dermatopontin interacts with transforming growth factor beta and enhances its biological activity. Biochem J. 1999; 337 ( Pt 3):537-41. | Article | PubMed Abstract | PubMed Full Text

5. Okamoto O, Suzuki Y, Kimura S and Shinkai H. Extracellular matrix 22kDa protein interacts with decorin core protein and is expressed in cutaneous fibrosis. J Biochem. 1996; 119:106-14. I Article I PubMed

6. Kato A, Okamoto O, Ishikawa K, Sumiyoshi H, Matsuo N, Yoshioka H, Nomizu M, Shimada T and Fujiwara S. Dermatopontin interacts with fibronectin, promotes fibronectin fibril formation, and enhances cell 
Coan et al. Musculoskeletal Biology 2014,

http://www.hoajonline.com/journals/pdf/2054-720X-1-2.pdf

doi: $10.7243 / 2054-720 \mathrm{X}-1-2$

adhesion. J Biol Chem. 2011; 286:14861-9. | Article | PubMed Abstract | PubMed Full Text

7. Takeda U, Utani A, Wu J, Adachi E, Koseki H, Taniguchi M, Matsumoto T, Ohashi T, Sato $M$ and Shinkai H. Targeted disruption of dermatopontin causes abnormal collagen fibrillogenesis. J Invest Dermatol. 2002; 119:678-83. | Article | PubMed

8. Takeuchi T, Suzuki M, Kumagai J, Kamijo T, Sakai M and Kitamura T. Extracellular matrix dermatopontin modulates prostate cell growth in vivo. J Endocrinol. 2006; 190:351-61. | Article | PubMed

9. Pochampally RR, Ylostalo J, Penfornis P, Matz RR, Smith JR and Prockop DJ. Histamine receptor $\mathrm{H} 1$ and dermatopontin: new downstream targets of the vitamin D receptor. J Bone Miner Res. 2007; 22:1338-49. | Article | PubMed

10. Coan HB, Teli $T$ and Booth $C$ et al. Cell-secreted matrices enhance osteogenesis in adipose-derived stem cells undergoing lineage specification. Journal of Biomimetics, Biomaterials and Tissue Engineering. 2012; 14:1-30. | Article

11. Livak KJ and Schmittgen TD. Analysis of relative gene expression data using real-time quantitative PCR and the 2(-Delta Delta $C(T)$ ) Method. Methods. 2001; 25:402-8. | Article | PubMed

12. Zuk PA, Zhu M, Ashjian P, De Ugarte DA, Huang JI, Mizuno H, Alfonso ZC, Fraser JK, Benhaim $P$ and Hedrick MH. Human adipose tissue is a source of multipotent stem cells. Mol Biol Cell. 2002; 13:4279-95. | Article | PubMed Abstract | PubMed Full Text

13. Philp D, Chen SS, Fitzgerald W, Orenstein J, Margolis L and Kleinman HK. Complex extracellular matrices promote tissue-specific stem cell differentiation. Stem Cells. 2005; 23:288-96. | Article | PubMed

14. Pham QP, Kasper FK, Scott Baggett L, Raphael RM, Jansen JA and Mikos AG. The influence of an in vitro generated bone-like extracellular matrix on osteoblastic gene expression of marrow stromal cells. Biomaterials. 2008; 29:2729-39. | Article | PubMed

15. Gamie Z, Tran GT, Vyzas G, Korres N, Heliotis M, Mantalaris A and Tsiridis E. Stem cells combined with bone graft substitutes in skeletal tissue engineering. Expert Opin Biol Ther. 2012; 12:713-29. | Article | PubMed

16. Jin HH, Kim DH, Kim TW, Shin KK, Jung JS, Park HC and Yoon SY. In vivo evaluation of porous hydroxyapatite/chitosan-alginate composite scaffolds for bone tissue engineering. Int J Biol Macromol. 2012; 51:1079-85. | Article | PubMed

17. Kang Y, Kim S, Bishop J, Khademhosseini A and Yang Y. The osteogenic differentiation of human bone marrow MSCs on HUVEC-derived ECM and beta-TCP scaffold. Biomaterials. 2012; 33:6998-7007. | Article | PubMed Abstract I PubMed Full Text

18. Abou Neel EA, Bozec L, Knowles JC, Syed O, Mudera V, Day R and Hyun JK. Collagen--emerging collagen based therapies hit the patient. Adv Drug Deliv Rev. 2013; 65:429-56. | Article | PubMed

19. Attia M, Santerre JP and Kandel RA. The response of annulus fibrosus cell to fibronectin-coated nanofibrous polyurethane-anionic dihydroxyoligomer scaffolds. Biomaterials. 2011; 32:450-60. | Article | PubMed

20. Wall I, Donos N, Carlqvist K, Jones F and Brett P. Modified titanium surfaces promote accelerated osteogenic differentiation of mesenchymal stromal cells in vitro. Bone. 2009; 45:17-26. | Article | PubMed

21. Ngiam M, Nguyen LT, Liao S, Chan CK and Ramakrishna S. Biomimetic nanostructured materials: potential regulators for osteogenesis? Ann Acad Med Singapore. 2011; 40:213-22. | Pdf | PubMed

22. Nguyen $\mathrm{TH}$ and Lee BT. In vitro and in vivo studies of rhBMP2-coated PS/PCL fibrous scaffolds for bone regeneration. J Biomed Mater Res A. 2013; 101:797-808. | Article | PubMed

23. Alford Al and Hankenson KD. Matricellular proteins: Extracellular modulators of bone development, remodeling, and regeneration. Bone. 2006; 38:749-57. | Article | PubMed

24. Bornstein $P$ and Sage EH. Matricellular proteins: extracellular modulators of cell function. Curr Opin Cell Biol. 2002; 14:608-16. | Article | PubMed
25. Bornstein P. Matricellular proteins: an overview. J Cell Commun Signal. 2009; 3:163-5. | Article | PubMed Abstract | PubMed Full Text

26. Chen CC and Lau LF. Functions and mechanisms of action of CCN matricellular proteins. Int J Biochem Cell Biol. 2009; 41:771-83. | Article | PubMed Abstract | PubMed Full Text

27. Leask $A$ and Abraham DJ. All in the CCN family: essential matricellular signaling modulators emerge from the bunker. J Cell Sci. 2006; 119:4803-10. | Article | PubMed

28. Tzen $\mathrm{CY}$ and Huang YW. Cloning of murine early quiescence-1 gene: the murine counterpart of dermatopontin gene can induce and be induced by cell quiescence. Exp Cell Res. 2004; 294:30-8. | Article | PubMed

29. Russell SB, Russell JD, Trupin KM, Gayden AE, Opalenik SR, Nanney LB, Broquist $A H$, Raju $L$ and Williams SM. Epigenetically altered wound healing in keloid fibroblasts. J Invest Dermatol. 2010; 130:2489-96. I Article | PubMed Abstract | PubMed Full Text

30. Sidgwick GP and Bayat A. Extracellular matrix molecules implicated in hypertrophic and keloid scarring. J Eur Acad Dermatol Venereol. 2012; 26:141-52. | Article | PubMed

31. Takemoto S, Murakami T, Kusachi S, Iwabu A, Hirohata S, Nakamura K, Sezaki S, Havashi J, Suezawa C, Ninomiya Y and Tsuji T. Increased expression of dermatopontin mRNA in the infarct zone of experimentally induced myocardial infarction in rats: comparison with decorin and type I collagen mRNAs. Basic Res Cardiol. 2002; 97:461-8. Article | PubMed

32. Catherino WH, Leppert PC, Stenmark MH, Payson M, Potlog-Nahari C, Nieman LK and Segars JH. Reduced dermatopontin expression is a molecular link between uterine leiomyomas and keloids. Genes Chromosomes Cancer. 2004; 40:204-17. | Article | PubMed

33. Kuroda $\mathrm{K}$, Okamoto $\mathrm{O}$ and Shinkai H. Dermatopontin expression is decreased in hypertrophic scar and systemic sclerosis skin fibroblasts and is regulated by transforming growth factor-beta1, interleukin-4, and matrix collagen. J Invest Dermatol. 1999; 112:706-10. | Article | PubMed

34. Scotti C, Tonnarelli B, Papadimitropoulos A, Scherberich A, Schaeren S, Schauerte A, Lopez-Rios J, Zeller R, Barbero A and Martin I. Recapitulation of endochondral bone formation using human adult mesenchymal stem cells as a paradigm for developmental engineering. Proc Natl Acad Sci U S A. 2010; 107:7251-6. | Article | PubMed Abstract I PubMed Full Text

35. Olivares-Navarrete R, Hyzy SL, Park JH, Dunn GR, Haithcock DA, Wasilewski CE, Boyan BD and Schwartz Z. Mediation of osteogenic differentiation of human mesenchymal stem cells on titanium surfaces by a Wnt-integrin feedback loop. Biomaterials. 2011; 32:6399-411. | Article | PubMed Abstract | PubMed Full Text

\section{Citation:}

Coan HB, Lively MO and Van Dyke ME.

Dermatopontin in the extracellular matrix enhances osteogenic differentiation of adipose-derived mesenchymal stem cells. Musculoskelet Biol. 2014; 1:2. http://dx.doi.org/10.7243/2054-720X-1-2 